\title{
Association of physician experience with higher prescription rate of anti-influenza agents in low-risk patients.
}

\author{
Koji Nakajima ${ }^{1}$, Hiroyuki Akebo ${ }^{1}$, Yukio Tsugihashi ${ }^{2}$, Hiroyasu Ishimaru ${ }^{1}$, and Ryuichi \\ Sada $^{1}$ \\ ${ }^{1}$ Public Interest Incorporated Foundation Tenri Hospital \\ ${ }^{2}$ Tenri Health Care University
}

August 17, 2020

\begin{abstract}
Rationale, aims, and objectives; During the influenza season, most patients suspected of having influenza undergo rapid influenza diagnostic tests (RIDTs) in Japan despite their low sensitivity. However, the physician's actual rationale for prescribing antivirals, besides the results of RIDTs, remains poorly understood. Our study sought to identify the role of clinical information and physicians' experience in the initiation of anti-influenza agents. Method; We retrospectively reviewed 380 patients who underwent RIDTs at the emergency department of our hospital from September 2018 to May 2019. Data regarding sex, age, etc., which could affect the decision of prescribing antivirals, were extracted from medical records. We performed logistic regression analysis to analyze the concurrent effect of potentially relevant clinical factors, results of RIDTs, and the physician's status on antiviral prescription. Results; Multivariable analysis revealed that a positive RIDT had the largest effect on antiviral prescription, followed by physician status, high regional influenza activity, and patients' presentation within 12 hours of symptom onset. Patient's age, comorbidities, and presentation after 48 hours of symptom onset were not associated with antiviral treatment. Physicians with more years of experience were significantly more likely to prescribe antivirals for patients with low risk of complications. Conclusions; Our findings revealed the physicians' rationale for initiating antiviral treatment and the discrepancy with guideline indications of antivirals, which is patient's age and comorbidities. Physicians, especially those with more than 3 years of experience, frequently prescribed antivirals for patients with low risk of complications; thus, educational interventions against this population could be useful to improve this situation.
\end{abstract}

Title:

Association of physician experience with higher prescription rate of anti-influenza agents in low-risk patients.

Running title: How do we prescribe anti-influenza drugs?

Author names and affiliations

Koji Nakajima, MD ${ }^{\mathrm{a}}$, Hiroyuki Akebo, MD ${ }^{\mathrm{a}}$, Yukio Tsugihashi, MD, MPH, MMM ${ }^{\mathrm{b}}$, Hiroyasu Ishimaru, $\mathrm{MD}^{\text {a }}$, Ryuichi Sada, MD ${ }^{\text {* }}$

${ }^{a}$ Department of General Internal Medicine, Tenri Hospital, Tenri, Nara, Japan

b Center for Healthcare Education, Tenri Health Care University, Tenri, Nara, Japan

* Corresponding author.

Ryuichi Sada

Department of General Internal Medicine, Tenri Hospital, 
200 Mishima-cho, Tenri-city, Nara, 632-8552, Japan.

Tel: +8174635611 ,

Fax: +81743631530 .

E-mail address: sadao@tenriyorozu.jp

Authorship statement:

All authors meet the ICMJE authorship criteria

Contributors

K.N.; Designed and analyzed the clinical research, R.S.; Supervised the research, All authors contributed to the interpretation of data and writing of the final manuscript. All authors approved the publish of the manuscript, and agreed to be accountable for all aspects of the work in ensuring that questions related to the accuracy or integrity of any part of the work are appropriately investigated and resolved.

Abstract

Rationale, aims, and objectives; During the influenza season, most patients suspected of having influenza undergo rapid influenza diagnostic tests (RIDTs) in Japan despite their low sensitivity. However, the physician's actual rationale for prescribing antivirals, besides the results of RIDTs, remains poorly understood. Our study sought to identify the role of clinical information and physicians' experience in the initiation of anti-influenza agents.

Method; We retrospectively reviewed 380 patients who underwent RIDTs at the emergency department of our hospital from September 2018 to May 2019. Data regarding sex, age, etc., which could affect the decision of prescribing antivirals, were extracted from medical records. We performed logistic regression analysis to analyze the concurrent effect of potentially relevant clinical factors, results of RIDTs, and the physician's status on antiviral prescription.

Results; Multivariable analysis revealed that a positive RIDT had the largest effect on antiviral prescription, followed by physician status, high regional influenza activity, and patients' presentation within 12 hours of symptom onset. Patient's age, comorbidities, and presentation after 48 hours of symptom onset were not associated with antiviral treatment. Physicians with more years of experience were significantly more likely to prescribe antivirals for patients with low risk of complications.

Conclusions; Our findings revealed the physicians' rationale for initiating antiviral treatment and the discrepancy with guideline indications of antivirals, which is patient's age and comorbidities. Physicians, especially those with more than 3 years of experience, frequently prescribed antivirals for patients with low risk of complications; thus, educational interventions against this population could be useful to improve this situation.

Keywords: Influenza; Influenza testing; Rapid influenza diagnostic testing; Antiviral Agents; Prescriptions; Medical Records

Introduction

Influenza is an acute respiratory viral infection that circulates all over the world. It is estimated to cause 3 to 5 million cases of severe illness and about 290,000 to 650,000 respiratory deaths every year. ${ }^{1}$ Prompt diagnosis and effective treatment are crucial, especially to those with high risks of complications, and the rapid influenza diagnostic tests (RIDTs) have been widely used in clinical practice for this purpose. ${ }^{2-4}$

Oseltamivir, the commonly used anti-influenza drug, is considered to shorten the duration of influenza symptoms by 16.8 hours; however, this drug has multiple side-effects including nausea and vomiting. ${ }^{5}$ Therefore, guidelines including those of the Infectious Diseases Society of America (IDSA) state that conservative management is adequate for the otherwise healthy and young population. ${ }^{6}$ RIDTs can be used to diagnose patients with high risks of influenza-related complications; however, two meta-analyses have recently revealed 
that the sensitivity of RIDT is $53-62 \%,,^{7,8}$ concluding that its false-negative rate is too high for clinical use during the endemic season.

In Japan, RIDTs are still almost always performed for patients with influenza-like illness during the influenza season, ${ }^{9}$ since they are considered a core tool in determining whether to initiate an antiviral treatment. This excessive use of RIDTs can also be inferred from the fact that, even with its relatively small population, Japan is one of the top consumers of anti-influenza agents in the world. ${ }^{9,10}$

Especially in a busy emergency department during the influenza season, physicians may have no choice but to rely on RIDT, whether they are aware of its low sensitivity or not. In that sense, clarifying the discrepancy between evidence and real-world practice is important, because it can lead to quality improvement of clinical practice. To the best of our knowledge, no such studies have been carried out in Japan, despite its strong dependence on RIDTs.

In this study, we aimed to detect the effect of each factor on the physician's rationale for prescribing antivirals and the discrepancy with current guideline recommendations at a Japanese hospital emergency department.

Methods

Study Setting and Design

This retrospective observational study was conducted at the emergency department of Tenri Hospital, an acute care 858-bed hospital in Nara, Japan. The emergency department of this teaching hospital accepts around 6000 ambulance transports and 15000 walk-in patients every year. Post graduate year (PGY) 1 residents and PGY-2 resident manage patients in the emergency department after gaining final approval from physicians above PGY-3. Those with more than 3 years of experience treat patients under their own discretion,

Medical records were reviewed for walk-in patients older than 15 years who had come to our emergency department from September 2018 to May 2019. During this period, a total of 695 patients had undergone RIDTs after a thorough clinical interview. Exclusion criteria were as follows: patients who had simultaneously undergone other tests, such as blood examination and chest x-ray for detecting more severe differential diagnosis than influenza: patients who had prolonged symptoms and had already visited a doctor within 2 weeks: patients who were transferred to another hospital and/or those who were prescribed antibiotics. After the application of these exclusion criteria, 380 patients were included in the study.

Patient information regarding comorbidities and presenting symptoms was obtained from the medical records written by the ER physicians. Comorbidities were defined as those related to the risks of influenza-related complications (Fig 1). ${ }^{6,11}$ Physician information was acquired regarding their years of experience. As of physicians with more than 3 years of experience, we also checked whether they specialize in internal medicine or in other fields (such as surgeons, radiologists, anesthesiologists etc.)

This study was approved by the institutional ethics committee (No. 1093), and written informed consent was waived because of the retrospective design.

Rapid influenza diagnostic tests

The RIDT that we used in this study was Quick Chaser Flu A, B@ (Mizuho Medy). ${ }^{12,13}$ This commercially available and widely used testing kit is based on immunochromatographic assay; a prior systematic review had concluded the overall sensitivity of this test to be $53^{\sim} 62 \%$ in clinical practice. ${ }^{7,8}$ Testing was performed as per the manufacturer's instructions.

Influenza Activity

In Japan, influenza surveillance is conducted as part of the National Epidemiological Surveillance of Infectious Diseases (NESID). The medical institution from each sentinel, selected by the prefectural government reports the weekly number of influenza cases to the area health center, and this data can be used to estimate the prefectural incidence of influenza. ${ }^{14}$ According to the NESID, the number of cases per sentinel exceeding 10.0 
per sentinel indicate an alert level. Therefore, we defined the influenza activity as high, when the number of cases per sentinel was greater than 10.0. ${ }^{14}$

Statistical Analysis

Continuous variables were presented as mean values with standard deviation and were examined using the Student's t-test. Nominal variables were compared using Pearson's chi-squared test. Multiple logistic regression analysis was used to identify factors that influence the administration of antivirals. Baseline variables with $\mathrm{p}<0.20$ in univariate analysis were included in the multivariable models. A p-value less than 0.05 was considered statistically significant. All statistical analysis was performed with IBM SPSS version 22.0 .

Results

During the study period, 695 patients were tested for influenza using RIDTs at the emergency department. 315 patients were excluded, including 270 patients who had undergone multiple tests, 39 patients who had a medical examination history within 2 weeks, 5 patients who were prescribed antibiotics, and 1 patient who was transferred. Three hundred-eighty patients were finally included in the analyses, $185(48.7 \%)$ with RIDT-positive, and 195 (51.3\%) with RIDT-negative. Among the patients with positive RIDT, 141 were prescribed antivirals while 44 were treated conservatively. Among the patients with negative RIDT, 20 were prescribed antivirals while 175 were treated conservatively.

Clinical information and its relation to antiviral prescription are shown in Table 1. Patient's symptom of cough $(\mathrm{p}=0.014)$, contact with another influenza patient $(\mathrm{p}<0.001)$, influenza activity in the region $(\mathrm{p}<0.001)$ differed significantly between groups treated with antivirals and groups treated conservatively. Patient's age, sex, comorbidities, or clinical symptoms besides cough did not differ significantly between groups treated with antivirals and groups treated conservatively.

Next, we conducted a multivariate analysis considering clinical factors that we assumed to be relevant to the prescription of antivirals in the univariate analysis (Tables 2, 3). Sex, age, comorbidities, the presence of cough, fever, results of RIDT, contact with influenza patient, influenza activity, and time from symptom onset were included in the model as covariates. The presence of fever was defined as body temperature above the cutoff value of 38.3 (100.9). Physician's status was also included in the analysis in terms of number of years of experience in Table 2, and specialty in Table 3. PGY 1-2 residents, PGY 3-5 residents, and PGY 6 or greater residents examined 257, 83, and 46 patients, respectively. Internists examined 93 patients while non-internists examined 36 patients. The patient's age, sex and the presence of comorbidities did not significantly affect the doctor's decision of antiviral prescription. Table 2 shows that the RIDT result had the largest influence on antiviral prescription (OR, 26.8; 95\% CI, 13.5-53.5; $\mathrm{P}<0.001)$, followed by high influenza activity (OR, 2.6; 95\% CI, 1.4-5.1; $\mathrm{P}=0.004$ ), and 12 hours within symptom onset (OR, 2.2; 95\% CI, 1.0-4.7; $\mathrm{P}=0.042$ ). Regarding the years of experience, physicians PGY3 or greater were more willing to prescribe antivirals than PGY 1-2 residents. Table 3 shows that among physicians greater that PGY 3, internists prescribed fewer antivirals than non-internists, though the difference was not statistically significant.

Finally, we hypothesized that doctors with more years of experience tend to prescribe antivirals to patients who do not require it according to the evidence-based recommendation. The guidelines recommend antiviral prescription for patients with high risk of complications, that is those who are "older than 65 years old" or "have comorbidities." Based on these recommendations, we divided the patients into "high-risk" and "low-risk." 87 patients were defined as "high-risk" and 293 patients were "low-risk." Then we compared the prescription rate of antivirals between these two groups based on the physician's years of experience (Table 4). Physicians with more than 3 years of experience prescribed significantly more antivirals to low-risk patients compared to those with less than 2 years of experience $(\mathrm{p}<0.001)$.

\section{Discussion}

In our observational study, we sought to confirm the clinical information that influences the antiviral prescription behavior of doctors. We found that antiviral prescription was associated with patient factors (the 
result of the RIDT and time from symptom onset), environmental factors (influenza activity in the local region), and physician factors (the doctor's years of experience). When we focused on the physician factors, having more years of experience led to more frequent administration of antivirals. This tendency was evident in patients with low risk of complications, although this population is not expected to benefit so much from antiviral treatment.

The relationship between the doctor's years of experience and specialty and their decision to prescribe antiviral agents has been implied in several prior studies ${ }^{15-17}$; however, all of them were survey-based researches and thus could not deny recall bias. On the other hand, our study was derived from the review of medical records, so we were able to establish a more reliable association between physicians' years of experience and prescription rate of antivirals.

The reason why physicians neglect evidence-based recommendations is said to be variable, including lack of awareness, lack of familiarity, and inertia of previous practice. ${ }^{18}$ Our results are consistent with previous findings that physicians tend to neglect evidence-based medicine when they are more experienced and have a dominant practice style. ${ }^{19,20}$ Moreover, although not significant, the fact that internists prescribed less than non-internists implied that lack of awareness could be a large contributing factor. This situation may be unique to Japan, where there are few acute care physicians, so non-internists must work at the emergency department. Nevertheless, the successful introduction of evidence-based medicine into real clinical practice is difficult and requires continuous feedback. ${ }^{20}$ Further studies on its barriers, and subsequent educational interventions, which is in this case against the high prescription rate of antivirals by physicians older than PGY 3 and non-internists, may improve the quality of practice pertaining to influenza. ${ }^{22}$

Finally, our study showed that the decision of antiviral prescription also depended on the time of the patient's arrival since symptom onset. Generally, RIDTs tend to produce false-negative results if conducted too early $\left(<12\right.$ hours), due to the low level of virus shedding at that time. ${ }^{23}$ In this study, we can infer that doctors acknowledged this limitation and were inclined toward prescribing antivirals to patients with negative RIDT if they presented themselves within 12 hours of symptom onset.

Several limitations should be acknowledged in this study. First, this study is based on medical records during a single influenza season at one hospital, so these findings may not apply to other influenza seasons and hospitals. Second, our study population had a restriction in that we enrolled only walk-in patients. However, patients brought in by ambulance are usually prescribed antivirals if they are suspected of having influenza. Also, selection bias may exist because this study only enrolled patients who had undergone RIDTs. However, in current practice, RIDTs are only used when influenza is suspected. Therefore, this population may well represent patients with clinical presentation suspectable of influenza. The pre-test probability could vary between each patient, but we took that into consideration by including factors other than RIDTs that may influence antiviral prescription in our analysis. Finally, we did not evaluate the patient outcome after the prescription of antivirals, so we could not accurately measure the effects of treatment in this study.

In conclusion, our study demonstrated that the clinician's decision to prescribe antivirals was influenced by the results of the RIDT, the clinician's years of experience, influenza activity, and time since symptom onset. Evidence-based indications for antivirals, such as patient's age, comorbidities, and presentation within 48 hours of symptom onset, were often neglected in real practice. We should promote educational interventions for older ER clinicians as well as PGY 1-2 residents to improve the quality of practice with regard to influenza.

\section{References}

1. World Health Organization (WHO). Influenza (seasonal) fact sheethttps://www.who.int/en/newsroom/fact-sheets/detail/influenza-(seasonal); 2018. Accessed May 27, 2020 2. Blaschke AJ, Shapiro DJ, Pavia AT, et al. A National Study of the Impact of Rapid Influenza Testing on Clinical Care in the Emergency Department. J Pediatric Infect Dis Soc. 2014;3(2):112-118. doi:10.1093/jpids/pit071. 3. Falsey AR, Murata Y, Walsh EE. Impact of rapid diagnosis on management of adults hospitalized with influenza. Arch Intern Med. 2007;167(4):354-360. doi:10.1001/archinte.167.4.ioi60207 4. Busson L, Mahadeb B, De Foor M, Van- 
denberg O, Hallin M. Contribution of a rapid influenza diagnostic test to manage hospitalized patients with suspected influenza. Diagn Microbiol Infect Dis. 2017;87(3):238-242. doi:10.1016/j.diagmicrobio.2016.11.015 5. Jefferson T, Jones M, Doshi P, Spencer EA, Onakpoya I, Heneghan CJ. Oseltamivir for influenza in adults and children: systematic review of clinical study reports and summary of regulatory comments. BMJ. 2014;348:g2545. Published 2014 Apr 9. doi:10.1136/bmj.g2545 6. Uyeki TM, Bernstein HH, Bradley JS, et al. Clinical Practice Guidelines by the Infectious Diseases Society of America: 2018 Update on Diagnosis, Treatment, Chemoprophylaxis, and Institutional Outbreak Management of Seasonal Influenzaa [published correction appears in Clin Infect Dis. 2019 May 2;68(10):1790]. Clin Infect Dis. 2019;68(6):e1e47. doi:10.1093/cid/ciy866 7. Chartrand C, Leeflang MM, Minion J, Brewer T, Pai M. Accuracy of rapid influenza diagnostic tests: a meta-analysis. Ann Intern Med. 2012;156(7):500-511. doi:10.7326/0003-4819156-7-201204030-00403 8. Merckx J, Wali R, Schiller I, et al. Diagnostic Accuracy of Novel and Traditional Rapid Tests for Influenza Infection Compared With Reverse Transcriptase Polymerase Chain Reaction: A Systematic Review and Meta-analysis. Ann Intern Med. 2017;167(6):394-409. doi:10.7326/M17-0848

9. Sugaya N. Widespread use of neuraminidase inhibitors in Japan. J Infect Chemother. 2011;17(5):595-601. doi:10.1007/s10156-011-0288-0

10. F. Hoffmann-La Roche Ltd. Media Release. Roche delivers solid results in 2014

http://www.roche.com/med-cor-2015-01-28-e.pdf; 2015. Accessed May 27, 2020.

11. Grohskopf LA, Sokolow LZ, Broder KR, Walter EB, Fry AM, Jernigan DB. Prevention and Control of Seasonal Influenza with Vaccines: Recommendations of the Advisory Committee on Immunization PracticesUnited States, 2018-19 Influenza Season. MMWR Recomm Rep. 2018;67(3):1-20. Published 2018 Aug 24. doi:10.15585/mmwr.rr6703a1

12. Sakai-Tagawa Y, Ozawa M, Yamada S, et al. Detection sensitivity of influenza rapid diagnostic tests. Microbiol Immunol. 2014;58(10):600-606. doi:10.1111/1348-0421.12185

13. Tokuno O, Fujiwara M, Nakajoh Y, et al. Comparison of detection sensitivity in rapid-diagnosis influenza virus kits. Kansenshogaku Zasshi. 2009;83(5):525-533. doi:10.11150/kansenshogakuzasshi.83.525

14. Hashimoto S, Kawado M, Murakami Y, et al. Number of sentinel medical institutions needed for estimating prefectural incidence in influenza surveillance in Japan. J Epidemiol. 2014;24(3):183-192. doi:10.2188/jea.je20130077

15. Katz MA, Lamias MJ, Shay DK, Uyeki TM. Use of rapid tests and antiviral medications for influenza among primary care providers in the United States. Influenza Other Respir Viruses. 2009;3(1):29-35. doi:10.1111/j.1750-2659.2009.00070.x

16. Mueller MR, Smith PJ, Baumbach JP, et al. Influenza testing and antiviral prescribing practices among emergency department clinicians in 9 states during the 2006 to 2007 influenza season. Ann Emerg Med. 2010;55(1):32-39. doi:10.1016/j.annemergmed.2009.09.019

17. Rothberg MB, Bonner AB, Rajab MH, Kim HS, Stechenberg BW, Rose DN. Effects of local variation, specialty, and beliefs on antiviral prescribing for influenza. Clin Infect Dis. 2006;42(1):95-99. doi:10.1086/498517

18. Cabana MD, Rand CS, Powe NR, et al. Why don't physicians follow clinical practice guidelines? A framework for improvement. JAMA. 1999;282(15):1458-1465. doi:10.1001/jama.282.15.1458

19. Halm EA, Atlas SJ, Borowsky LH, et al. Understanding physician adherence with a pneumonia practice guideline: effects of patient, system, and physician factors. Arch Intern Med. 2000;160(1):98-104. doi:10.1001/archinte.160.1.98

20. Halm EA, Atlas SJ, Borowsky LH, Benzer TI, Singer DE. Change in physician knowledge and attitudes after implementation of a pneumonia practice guideline. J Gen Intern Med. 1999;14(11):688-694. doi:10.1046/j.1525-1497.1999.03469.x 
21. Grimshaw JM, Russell IT. Effect of clinical guidelines on medical practice: a systematic review of rigorous evaluations. Lancet. 1993;342(8883):1317-1322. doi:10.1016/0140-6736(93)92244-n

22. Lagerløv P, Loeb M, Andrew M, Hjortdahl P. Improving doctors' prescribing behaviour through reflection on guidelines and prescription feedback: a randomised controlled study. Qual Health Care. 2000;9(3):159-165. doi:10.1136/qhc.9.3.159

23. Tanei M, Yokokawa H, Murai K, et al. Factors influencing the diagnostic accuracy of the rapid influenza antigen detection test (RIADT): a cross-sectional study. BMJ Open. 2014;4(1):e003885. Published 2014 Jan 2. doi:10.1136/bmjopen-2013-003885

Acknowledgments:None. No financial nor material support.

Conflict of interest:None

Table 1 Clinical information and its association with antiviral prescription

\begin{tabular}{|c|c|c|c|}
\hline & Antiviral prescription & Antiviral prescription & \\
\hline & Yes $(n=166)$ & No $(n=214)$ & $\mathrm{p}$ \\
\hline $\mathrm{RIDT}^{+}$positive $(\%)$ & 84.9 & 20.6 & $<0.001^{* * *}$ \\
\hline Sex (male), n (\%) & 52.4 & 52.8 & 0.94 \\
\hline Age $(y r)$, mean \pm SD & $35.2 \pm 18.7$ & $35.1 \pm 17.4$ & 0.97 \\
\hline $\begin{array}{l}\text { Body temperature }(), \text { mean } \pm \text { SD } \\
\text { Symptoms, } \mathrm{n}(\%)\end{array}$ & $38.2 \pm 0.98$ & $38.1 \pm 0.88$ & $0.099^{*}$ \\
\hline Cough & 71.1 & 58.9 & $0.014^{* *}$ \\
\hline Myalgias & 13.3 & 15 & 0.64 \\
\hline Chills or Sweats & 35.5 & 38.3 & 0.58 \\
\hline High Risks for Complications, n (\%) & & & \\
\hline Age $>=65$ & 10.8 & 8.4 & 0.42 \\
\hline Comorbidities & 15.7 & 19.6 & 0.32 \\
\hline Epidemiologic Information, n (\%) & & & \\
\hline Contact with influenza patient & 50 & 32.2 & $<0.001^{* * *}$ \\
\hline High Influenza activity & 64.5 & 43 & $<0.001^{* * *}$ \\
\hline Time from symptom onset, n (\%) & & & 0.13 \\
\hline$<12$ hours & 25.3 & 28 & \\
\hline 12 hours $\sim 48$ hours & 63.3 & 54.2 & \\
\hline$>=48$ hours & 11.4 & 17.8 & \\
\hline
\end{tabular}

Data are mean \pm SD or $\%$

${ }^{+}$RIDT, rapid influenza diagnostic test

${ }^{*} \mathrm{p}<0.1 ;{ }^{* *} \mathrm{p}<0.05 ;{ }^{* * *} \mathrm{p}<0.01$

Table 2 Multivariate factors that influence a physician's decision to prescribe antiviral drugs according to the physician's status classified by years of experience.

\begin{tabular}{llll}
\hline & Odds ratio & $95 \%$ CI & $\mathrm{p}$ \\
\hline Sex, male & 1.3 & $0.70-2.3$ & 0.43 \\
Age $>=65$ & 1.7 & $0.60-4.7$ & 0.33 \\
Comorbidities & 0.82 & $0.38-1.7$ & 0.59 \\
RIDT $^{+}$positive & 26.8 & $13.5-53.5$ & $<0.001^{* * *}$ \\
Cough & 0.85 & $0.43-1.7$ & 0.63 \\
Fever & 1.2 & $0.66-2.1$ & 0.56
\end{tabular}




\begin{tabular}{|c|c|c|c|}
\hline & Odds ratio & $95 \%$ CI & $\mathrm{p}$ \\
\hline Contact with influenza patient & 1.4 & $0.77-2.7$ & 0.26 \\
\hline High Influenza activity & 2.6 & $1.4-5.1$ & $0.004^{* * *}$ \\
\hline \multicolumn{4}{|l|}{ Time from symptom onset } \\
\hline 12 hours $\sim 48$ hours & 1 (Reference) & 1 (Reference) & \\
\hline$<12$ hours & 2.2 & $1.0-4.7$ & $0.042^{* *}$ \\
\hline$>=48$ hours & 0.86 & $0.35-2.1$ & 0.75 \\
\hline \multicolumn{4}{|l|}{ ER Physician } \\
\hline $\mathrm{PGY}^{++} 1-2$ resident & 1 (Reference) & 1 (Reference) & \\
\hline $\mathrm{PGY}^{++} 3-5$ resident & 3.9 & $1.8-8.3$ & $<0.001^{* * *}$ \\
\hline $\mathrm{PGY}^{++} 6$ or greater & 5.3 & $2.0-14.4$ & $0.001^{* * *}$ \\
\hline
\end{tabular}

+ RIDT, Rapid influenza diagnostic test; ${ }^{++}$PGY, post-graduate year

${ }^{*} \mathrm{p}<0.1 ;{ }^{* *} \mathrm{p}<0.05 ;{ }^{* * *} \mathrm{p}<0.01$

Table 3 Multivariate factors that influence physician's decision of antiviral prescription according to the physician's status classified by specialty.

\begin{tabular}{llll}
\hline & Odds ratio & $95 \%$ CI & $\mathrm{p}$ \\
\hline Sex, male & 1.3 & $0.71-2.4$ & 0.4 \\
Age $>=65$ & 1.8 & $0.63-5.0$ & 0.28 \\
Comorbidities & 0.78 & $0.37-1.6$ & 0.51 \\
RIDT $^{+}$positive & 28.4 & $14.1-57.1$ & $<0.001^{* * *}$ \\
Cough $_{\text {Fever }}$ & 0.87 & $0.44-1.7$ & 0.69 \\
Contact with influenza patient & 1.2 & $0.66-2.1$ & 0.56 \\
High Influenza activity & 1.4 & $0.75-2.6$ & 0.29 \\
Time from symptom onset & 2.5 & $1.3-4.8$ & $0.005^{* * *}$ \\
12hours 48 hours & 1 (Reference) & 1 (Reference) \\
$<12$ hours & 2.2 & $1.0-4.7$ & $0.046^{* *}$ \\
$>=48$ hours & 0.86 & $0.35-2.1$ & 0.75 \\
ER Physician & & & \\
PGY ${ }^{++}$1-2 resident & 1 (Reference) & 1 (Reference) & \\
Internists & 3.7 & $1.8-7.7$ & $<0.001^{* * *}$ \\
Non-Internists & 6.3 & $2.3-17.8$ & $<0.001^{* * *}$ \\
\hline
\end{tabular}

+ RIDT, Rapid influenza diagnostic test; ${ }^{++}$PGY, post-graduate year

${ }^{*} \mathrm{p}<0.1 ;{ }^{* *} \mathrm{p}<0.05 ;{ }^{* * *} \mathrm{p}<0.01$

Table 4 Prescription rate based on the physician's status and patients' risk of complications

\begin{tabular}{lllll}
\hline & High-risk patients & $\mathrm{p}$ & Low-risk patients & $\mathrm{p}$ \\
\hline PGY 1-2 resident & $22 / 55$ & 0.88 & $72 / 196$ & $<0.01 * * *$ \\
PGY 3-5 resident & $8 / 18$ & & $37 / 65$ & \\
PGY 6 or greater & $5 / 14$ & & $22 / 32$ & \\
\hline
\end{tabular}


Data are $\%$

PGY, post-graduate year

${ }^{*} \mathrm{p}<0.1 ;{ }^{*} \mathrm{p}<0.05 ; * * * \mathrm{p}<0.01$

Figure legend

Figure 1 Comorbidities considered risk factors for influenza-related complications

Figure 2 Flowchart of patient inclusion criteria

RIDT, rapid influenza diagnostic test

\section{Hosted file}

Figures 2020.08.16.docx available at https://authorea.com/users/351487/articles/476088association-of-physician-experience-with-higher-prescription-rate-of-anti-influenzaagents-in-low-risk-patients 